\title{
SOME INEQUALITIES FOR THE SPECTRAL RADIUS OF THE HADAMARD PRODUCT OF TWO NONNEGATIVE MATRICES
}

\author{
GuAnghui CHEng And Xi RaO
}

Abstract. In this paper, we propose some sharper upper bounds for the spectral radius of the Hadamard product of two nonnegative matrices. The results involve the directed graph of the Hadamard product of associated matrices.

Mathematics subject classification (2010): 15A42, 15A18.

Keywords and phrases: The Hadamard product, the spectral radius, nonnegative matrix, inverse $M$ matrix, directed graph.

\section{REFERENCES}

[1] A. Berman and R. J. Plemmons, Nonnegative Matrices in the Mathematical Sciences, Classics in Applied Mathematics, 9, SIAM, Philadelphia, 1994.

[2] S. C. CHEN, A lower bound for the minimum eigenvalue of the Hadamard product of matrices, Linear Algebra. Appl., 378, (2004), 159-166.

[3] G. H. Cheng, X. Y. Cheng, T. Z. Huang And T. Y. TAM, Some bounds for the spectral radius of the Hadamard product of matrices, Applied Math. E-notes, 5, (2005), 202-209.

[4] R. A. Horn And C. R. Johnson, Matrix Analysis, Cambridge University Press, 1985.

[5] R. A. Horn And C. R. Johnson, Topics in Matrix Analysis, Cambridge University Press, 1991.

[6] R. HuAng, Some inequalities for the Hadamard product and the Fan product of matrices, Linear Algebra. Appl., 428, (2008), 1551-1559.

[7] L. Y. Kolotilina, Bounds for the Perron root, singularity/nonsingularity conditions, and eigenvalue inclusion sets, Numer. Algorithm, 42, (2006), 247-280.

[8] Q. B. Liu And G. L. Chen, On two inequalities for the Hadamard product and the Fan product of matrices, Linear Algebra. Appl., 431, (2009), 974-984.

[9] Y. Z. SONG, On an inequality for the Hadamard product of an $M$-matrix and its inverse, Linear Algebra. Appl., 305, (2000), 99-105.

[10] Y. M. Zheng AND R. Q. CUI, Upper bound of the spectral radius of the Hadamard product of nonnegative matrices, Journal of Henan Polytechnic University (Natural science), 29, (4) (2010), 543546. 